\title{
Alcohol intake, drinking patterns, and prostate cancer risk and mortality : a 30-year prospective cohort study of Finnish twins
}

\section{Dickerman, Barbra A.}

2016-09

Dickerman , B A, Markt , S C , Koskenvuo , M , Pukkala , E , Mucci , L A \& Kaprio , J 2016 , ' Alcohol intake, drinking patterns, and prostate cancer risk and mortality : a 30-year prospective cohort study of Finnish twins ', Cancer Causes \& Control , vol. 27 , no. 9 , pp. 1049-1058 . https://doi.org/10.1007/s10552-016-0778-6

http://hdl.handle.net/10138/229843

https://doi.org/10.1007/s10552-016-0778-6

publishedVersion

Downloaded from Helda, University of Helsinki institutional repository.

This is an electronic reprint of the original article.

This reprint may differ from the original in pagination and typographic detail.

Please cite the original version. 


\title{
Alcohol intake, drinking patterns, and prostate cancer risk and mortality: a 30-year prospective cohort study of Finnish twins
}

\author{
Barbra A. Dickerman ${ }^{1}$ - Sarah Coseo Markt ${ }^{1} \cdot$ Markku Koskenvuo $^{2}$. \\ Eero Pukkala $^{3,4}$ - Lorelei A. Mucci ${ }^{1}$ Jaakko Kaprio ${ }^{2,5,6}$
}

Received: 26 January 2016/ Accepted: 10 June 2016/Published online: 28 June 2016

(C) Springer International Publishing Switzerland 2016

\begin{abstract}
Purpose Alcohol intake may be associated with cancer risk, but epidemiologic evidence for prostate cancer is inconsistent. We aimed to prospectively investigate the association between midlife alcohol intake and drinking patterns with future prostate cancer risk and mortality in a population-based cohort of Finnish twins.

Methods Data were drawn from the Older Finnish Twin Cohort and included 11,372 twins followed from 1981 to 2012. Alcohol consumption was assessed by questionnaires administered at two time points over follow-up. Over the study period, 601 incident cases of prostate cancer and 110 deaths from prostate cancer occurred. Cox regression was used to evaluate associations between weekly alcohol
\end{abstract}

Electronic supplementary material The online version of this article (doi:10.1007/s10552-016-0778-6) contains supplementary material, which is available to authorized users.

Jaakko Kaprio

jaakko.kaprio@helsinki.fi

1 Department of Epidemiology, Harvard T.H. Chan School of Public Health, Boston, MA, USA

2 Department of Public Health, University of Helsinki, Mannerheimintie 172, P.O. Box 41, 00014 Helsinki, Finland

3 Finnish Cancer Registry, Institute for Statistical and Epidemiological Cancer Research, Unioninkatu 22, 00130 Helsinki, Finland

4 School of Health Sciences, University of Tampere, 33014 Tampere, Finland

5 Department of Health, National Institute for Health and Welfare, Mannerheimintie 166, P.O. Box 30, 00300 Helsinki, Finland

6 Institute for Molecular Medicine (FIMM), University of Helsinki, Tukholmankatu 8, P.O. Box 20, 00014 Helsinki, Finland intake and binge drinking patterns with prostate cancer risk and prostate cancer-specific mortality. Within-pair co-twin analyses were performed to control for potential confounding by shared genetic and early environmental factors.

Results Compared to light drinkers ( $\leq 3$ drinks/week; nonabstainers), heavy drinkers ( $>14$ drinks/week) were at a 1.46-fold higher risk (HR 1.46; $95 \%$ CI 1.12, 1.91) of prostate cancer, adjusting for important confounders. Among current drinkers, binge drinkers were at a significantly increased risk of prostate cancer (HR 1.28; $95 \% \mathrm{CI}$ $1.06,1.55)$ compared to non-binge drinkers. Abstainers were at a 1.90-fold higher risk (HR 1.90; $95 \%$ CI 1.04, $3.47)$ of prostate cancer-specific mortality compared to light drinkers, but no other significant associations for mortality were found. Co-twin analyses suggested that alcohol consumption may be associated with prostate cancer risk independent of early environmental and genetic factors.

Conclusion Heavy regular alcohol consumption and binge drinking patterns may be associated with increased prostate cancer risk, while abstinence may be associated with increased risk of prostate cancer-specific mortality compared to light alcohol consumption.

Keywords Alcohol - Binge drinking - Prospective cohort study $\cdot$ Prostate cancer $\cdot$ Twins

\section{Introduction}

Alcohol is an established risk factor for cancers of the oral cavity, pharynx, larynx, esophagus, liver, colorectum, and female breast, but evidence for an association between alcohol and prostate cancer is inconsistent [1-3]. There is 
biologic rationale that alcohol or its metabolites (e.g., acetaldehyde) could increase prostate cancer risk by acting as a carcinogen or by modulating risk from other carcinogens [4-7]. Since alcohol consumption is a common and modifiable lifestyle factor, even a modest association with prostate cancer risk would be of public health significance.

Over 70 epidemiologic studies have explored the relationship between alcohol and prostate cancer, but findings have been mixed and critical gaps remain [reviewed in 1; $8-11]$. While most studies have reported evidence of little to no association between alcohol consumption within normal range and prostate cancer risk overall, [1, 11-13] some have suggested an elevated risk among heavy drinkers [14-22]. The risk associated with heavy regular alcohol intake as well as binge drinking patterns deserves further exploration. The influence of midlife alcohol consumption on later prostate cancer risk is also of great interest given the long latency of prostate cancer and biologic plausibility that alcohol may act as a promoter of this disease [5]. Finally, prospective study designs and unique analytic methods are needed to minimize the possibility that the modest associations that have been detected could be explained by unknown confounders or biases inherent to many observational studies.

We aimed to prospectively investigate the influence of midlife alcohol intake and drinking patterns on prostate cancer risk later in life in the Older Finnish Twin Cohort, a population-based cohort of male twins with a median of 30 years of follow-up data, a wide range of alcohol consumption assessed at two time points, and a high prevalence of reported binge drinking. In addition to examining the risk of prostate cancer diagnosis, we explored the risk of prostate cancer-specific mortality, which reflects the most clinically aggressive disease. A detailed examination of these associations has never before been explored in a prospective study of male twins-a setting that allows for the application of powerful analytic methods to control for potential familial confounding (genetics and shared early environment). We hypothesize that high levels of average weekly alcohol consumption and binge drinking behavior are associated with increased prostate cancer risk.

\section{Materials and methods}

\section{Study population}

This study was nested within the Older Finnish Twin Cohort, consisting of all Finnish same-sex twin pairs born before 1958 with both co-twins alive in 1975. Twin pairs were selected from the Central Population Registry of Finland in 1974. Twin zygosity was determined by a validated questionnaire shown to accurately classify $>93 \%$ of twin pairs as monozygotic (MZ) or dizygotic (DZ) [23]. This survey included questions on whether twins resembled one another and whether strangers would confuse them in childhood and was validated against polymorphic blood markers. Questionnaires were mailed to participants in 1975 and 1981, with response rates of 89 and $84 \%$, respectively. They contained questions on lifestyle factors such as alcohol consumption, smoking, and physical activity, in addition to comprehensive questions on socio-demographic, psychosocial, and health factors. A total of 11,372 male twin individuals responded to both the 1975 and 1981 questionnaires and were free of prostate cancer in 1981. Among these men, there were 1,290 monozygotic (MZ) and 2,858 dizygotic (DZ) twin pairs, in addition to 442 twin pairs of uncertain zygosity. In addition, there were 423 singleton twins (twins without their co-twin in the study) of unknown zygosity, 457 singleton MZ twins, and 1,312 singleton DZ twins. Of these 11,372 men, 11,363 provided information on alcohol intake (regular consumption in 1975 and 1981 and/or binge drinking in 1981). A total of 11,352 men contributed to the regular alcohol consumption analyses, and 11,164 men contributed to the binge drinking analyses $(11,153$ of whom also had data on regular alcohol consumption and 11 of whom had data on binge drinking only). The mean age ( \pm standard deviation) of participants at the time of study entry was 40.1 years $( \pm 12.5)$.

The study was approved by the ethical committee of the Hjelt Institute, Faculty of Medicine, University of Helsinki. Permission for linkage of the cancer registry data was provided by the National Institute for Health and Welfare, Helsinki, Finland. Informed consent was obtained from all respondents.

\section{Exposure assessment}

Given the long latency of prostate cancer, we were interested in the influence of midlife alcohol intake on prostate cancer risk and mortality later in life. Information on alcohol intake was obtained on both the 1975 and 1981 questionnaires by asking respondents to report the quantity of beer and wine consumed in an average week and the quantity of spirits consumed in an average month. The amount of alcohol consumed was measured on 3 separate scales (one for each type of alcoholic beverage), with the upper limits defined as consuming $\geq 48$ bottles of beer/ week, $\geq 10$ bottles of wine/week, and $\geq 20$ bottles of spirits/month (defined as any alcohol stronger than fortified wine). In 1975 and 1981 in Finland, beer bottles were almost exclusively $0.33 \mathrm{~L}$, wine bottles were generally $0.75 \mathrm{~L}$, and vodka (the most common spirit being used) was sold in $0.5 \mathrm{~L}$ bottles. Reported consumption of each 
beverage type was converted into grams of absolute alcohol and summed to create total alcohol intake in grams/ week. We averaged estimates from the 1975 and 1981 questionnaires to estimate average alcohol intake in units of grams/week. We then converted this average to a value of drinks/week, defining one drink as 12 grams of alcohol. Current drinkers were classified as light (0.01-3 drinks/week), moderate (3.01-14 drinks/week), or heavy ( $>14.01$ drinks/week) drinkers, per National Institute on Alcohol Abuse and Alcoholism (NIHAAA) guidelines [24].

We distinguished lifetime abstainers as those who reported no alcohol intake on both the 1975 and 1981 questionnaires from former drinkers defined as abstinent on both the 1975 and 1981 questionnaires, but reported a history of drinking prior to the 1975 questionnaire. These two groups, in addition to a small group $(n=115)$ reporting abstinence in 1975 or 1981 and missing alcohol use data in 1975 or 1981, were collapsed into an "abstainers" category that referred to current nondrinkers.

Binge drinkers were those who responded that they had consumed either: $>5$ bottles of beer, 1 bottle of wine, or 4 drinks ( $\geq 18$ milliliters of spirits) on the same occasion at least once a month during the preceding year on the 1981 questionnaire.

\section{Outcome ascertainment}

Follow-up of the twin cohort was achieved through linkage with national health registers in Finland. Data on prostate cancer incidence (ICD code 185) were obtained through record linkage to the Finnish Cancer Registry, where $100 \%$ of registered cases are histologically verified. Cause-of-death data were obtained through linkage with Statistics Finland. All of those who died from prostate cancer had a diagnosis of prostate cancer prior to death in the Finnish Cancer Registry. Data on emigration and vital status were obtained through linkage to the Population Register Center of Finland. Data from all registries were linked to Finnish Twin Cohort data using unique personal identity codes assigned to every permanent resident of Finland. The completeness of these registers allows for essentially complete disease follow-up.

\section{Statistical analysis}

Descriptive analyses (frequencies and means) were calculated to characterize the study population and examine differences in potential confounders across categories of average weekly alcohol consumption. Our primary exposures of interest were average weekly alcohol consumption and binge drinking among current drinkers. Cox proportional hazard models were used to estimate age-adjusted hazard ratios (HRs) with $95 \%$ confidence intervals (CIs) for the outcomes of prostate cancer incidence and prostate cancer-specific mortality. Each participant's date of entry was defined as his exact age when the 1981 questionnaire was returned. Participants were followed prospectively through 31 December 2012 for the occurrence of prostate cancer, death from any cause, or emigration. Log-log plots of survival curves of average weekly alcohol consumption and binge drinking status were used to verify that the curves were parallel and the proportional hazards assumption was not violated. Age was the underlying time metameter. Our final multivariable models were adjusted for potential confounding variables: BMI (continuous), smoking (never, occasional, former, current), social class (upper white collar, lower white collar, skilled worker, unskilled worker, farmer, other), education ( $<6$ years, 6 years, middle school, high school, or more), and physical activity (sedentary, occasional exerciser, conditioning exerciser). Risk estimates for alcohol use remained stable whether social class and education were included together in the model or not (data not shown). Due to the dependent nature of our study population (twin pairs), standard errors and CIs were adjusted for possible withinpair correlations using robust variance estimators.

We further performed co-twin analyses to investigate the association between alcohol consumption and prostate cancer risk within twin pairs discordant for alcohol intake category and time to event (i.e., time to prostate cancer diagnosis among twin pairs concordant for prostate cancer outcome, and time to event vs. death or end of follow-up among twin pairs discordant for prostate cancer outcome; see Supplementary Figure 1). These Cox models were stratified on twin pairs, allowing each twin pair to have its own baseline hazard. This serves as a powerful approach to account for familial confounding (genetics and shared early environment) when assessing twins discordant for alcohol consumption and prostate cancer outcomes. If the association between alcohol consumption and prostate cancer were rooted in familial factors, one would expect an association to exist between twin pairs (in individual-based analyses of the entire cohort) but not within twin pairs raised in the same household [25]. On the other hand, if the association were rooted in genetic factors, then the association should be present within DZ twin pairs (sharing on average $50 \%$ of their segregating genes) but not within MZ twin pairs (sharing $100 \%$ of their genome sequence). Lastly, if the association were rooted in non-familial environmental factors (independent of genetics and shared early environment), then the association should be present within both DZ and MZ twin pairs. Non-familial environmental factors include all environmental factors unique to twin individuals as well as direct causal associations between alcohol consumption and prostate cancer. 
We performed a predefined subgroup analysis to explore a potential interaction between average weekly alcohol consumption and smoking status (never, former, occasional, daily). The presence of effect modification was assessed by entering product terms of average weekly alcohol intake and smoking status categories into the model and estimating likelihood ratio tests comparing models with and without the interaction terms.

Finally, we explored the association between our measures of alcohol consumption and alcohol-related causes of death (alcoholism, alcoholic liver disease, and alcoholic pancreatitis). We calculated the relative proportion of deaths due to alcohol-related causes for each category of average alcohol consumption. We also performed Cox regression to examine associations between average weekly alcohol consumption and risk of alcohol-related death. Because alcohol-related causes of death require heavy alcohol use for a long period of time, this approach enabled us to explore the stability of alcohol consumption category over time.

All statistical tests were two-sided, and $p$ values $<0.05$ were considered statistically significant. All statistical analyses were performed using Stata version 13.1 (Stata Corporation, College Station, TX, USA).

\section{Results}

Over a median of 30 years of follow-up, 601 incident cases of prostate cancer and 110 deaths from prostate cancer occurred. The mean age ( \pm standard deviation) of participants at the time of prostate cancer diagnosis was 69.9 years $( \pm 8.9)$.

Baseline characteristics of participants are displayed in Table 1 by category of average alcohol consumption (drinks/week). The distribution of average weekly alcohol consumption in this study population was as follows: $7 \%$ abstainers, $31 \%$ light drinkers, $46 \%$ moderate drinkers, and $17 \%$ heavy drinkers. Light drinkers consumed a mean of 1.7 drinks/week, moderate drinkers consumed a mean of 7.1 drinks/week, and heavy drinkers consumed a mean of 24.1 drinks/week. Heavy drinkers were younger, had a higher BMI, and were more likely to be current smokers and binge drinkers compared to those in other drinking categories.

\section{Prostate cancer diagnosis}

In the overall study population, average weekly alcohol consumption (continuous) was significantly associated with prostate cancer risk in age-adjusted models, whereby each additional drink consumed per day was associated with a $10 \%$ increase in prostate cancer risk (HR 1.10; $95 \%$ CI
$1.05,1.15)$. Exclusion of abstainers did not influence the relative risk estimate (data not shown).

Modeling average weekly alcohol consumption as a categorical variable, we found a positive association with prostate cancer risk among moderate (HR 1.20; $95 \%$ CI $0.99,1.46$ ) and heavy drinkers (HR $1.46 ; 95 \%$ CI 1.12 , 1.91) compared to light drinkers in multivariable models (Table 2). Results using abstinent men as the reference group are displayed in Supplementary Table 1. Among current drinkers, binge drinkers were at a significantly increased risk of prostate cancer compared to non-binge drinkers in age-adjusted (HR 1.35; $95 \%$ CI 1.12, 1.61) and multivariable (HR 1.28; $95 \%$ CI 1.06, 1.55) models (Table 2).

Among the twin pairs, 354 twin pairs were discordant for prostate cancer outcome, such that one twin was a prostate cancer case and his co-twin brother was not. Of these 354 pairs, 195 were also discordant for average weekly alcohol consumption category (Table 3) and 102 were discordant for binge drinking status. Supplementary Tables 2 and 3 display counts of exposure and outcome concordance for DZ and MZ twin pairs, respectively. Prostate cancer risk estimates obtained from pairwise twin analyses were consistently higher than those obtained from individual-based analyses. Within twin pairs, abstainers were at the highest risk of prostate cancer compared to their light drinker brothers (HR 2.98; $95 \%$ CI 1.35, 6.60) (Table 4). This elevated risk remained statistically significant among DZ twin pairs (HR 3.80; $95 \%$ CI 1.36, 20.6) but not among MZ twin pairs (HR 2.85; $95 \%$ CI 0.67 , 12.1). Pairwise analysis results using abstinent men as the reference group are displayed in Supplementary Table 4. Within discordant twin pairs, binge drinking was suggestively, but not significantly, associated with an increased risk of prostate cancer (HR 1.47; $95 \%$ CI 0.96, 2.25) (Table 5). In sensitivity analyses, pairwise analyses restricted to twin pairs discordant for prostate cancer outcome produced similar estimates but with wider confidence intervals (data not shown).

\section{Prostate cancer-specific mortality}

In the overall study population, average weekly alcohol consumption (continuous) was positively associated with prostate cancer mortality (HR 1.06; $95 \%$ CI 0.91, 1.23), although not statistically significant (data not shown).

Modeling average weekly alcohol consumption as a categorical variable, abstainers were at a significantly higher risk of death from prostate cancer (HR 1.90; $95 \%$ CI 1.04, 3.47) compared to light drinkers (Table 2). We decomposed this group of current nondrinkers (in both 1975 and 1981) into lifetime abstainers $(n=588)$ and former drinkers (i.e., prior to $1975 ; n=78$ ) to assess the 
Table 1 Baseline characteristics across categories of average alcohol consumption in drinks/week (average of 1975 and 1981 ), Older Finnish Twin Cohort, 1981-2012

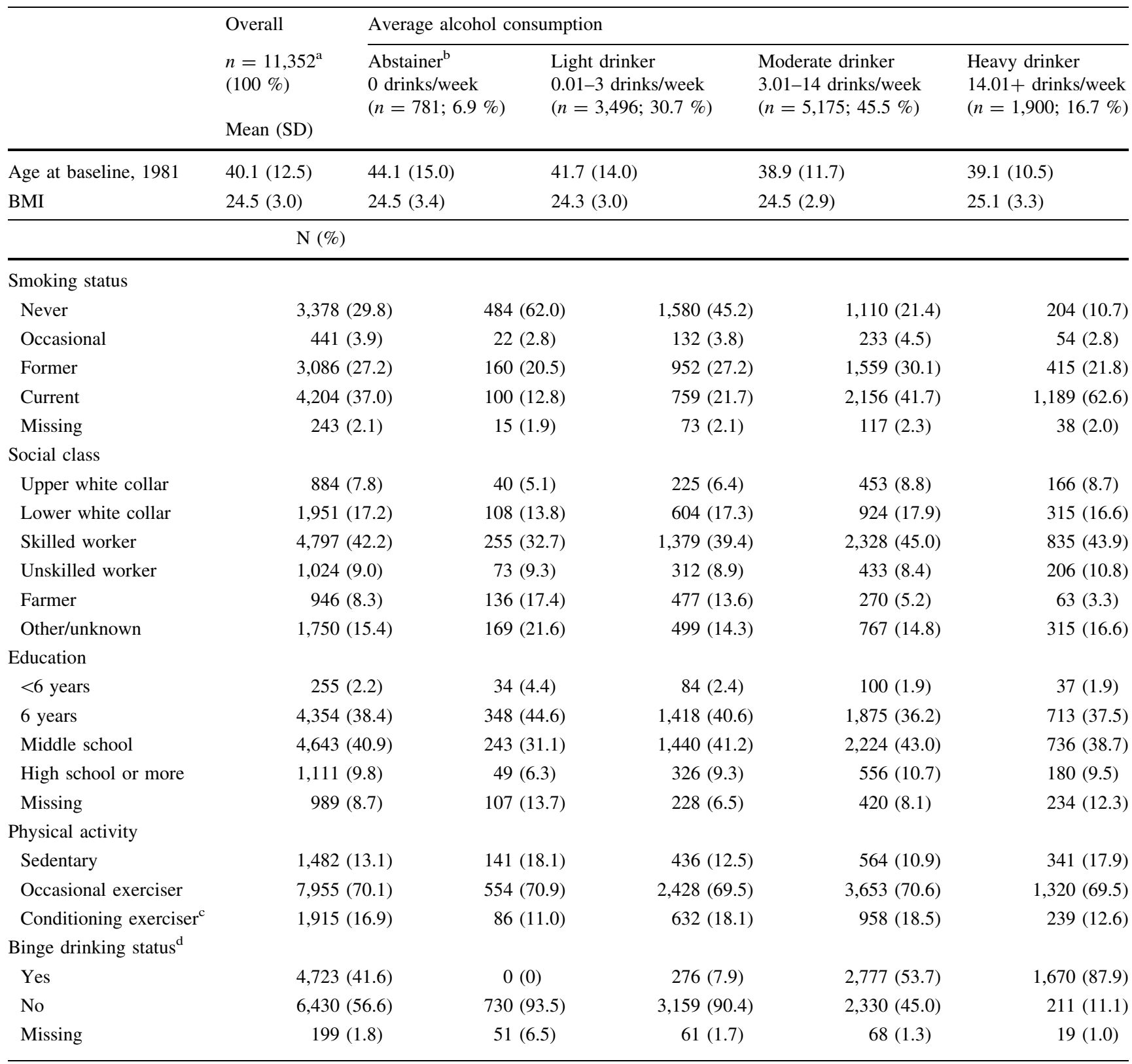

$B M I$ body mass index, calculated from self-reported height and weight; $M E T$ metabolic equivalents

${ }^{a} \mathrm{~N}=11,352$ participants had data on average alcohol consumption category

b Abstainers refer to lifetime abstainers, former drinkers (those who were abstinent in both 1975 and 1981 but reported a history of drinking prior to the 1975 questionnaire), as well as participants who reported abstinence in 1975 or 1981 and were missing alcohol use data in 1975 or 1981

${ }^{\mathrm{c}}$ Conditioning exerciser refers to those reporting exercising at least 6 times per month for a mean duration of at least 30 min and with a mean intensity corresponding to at least vigorous walking to jogging

${ }^{\mathrm{d}}$ Binge drinking status refers to reported consumption of $>5$ bottles of beer, 1 bottle of wine, or 4 drinks ( $\geq 18 \mathrm{~mL}$ of spirits) on the same occasion at least once a month during the preceding year

risk associated with each distinct subgroup. Lifetime abstainers were at a significantly increased risk of prostate cancer-specific mortality (HR 2.18; $95 \%$ CI 1.19, 3.99), and former drinkers were at a nonsignificantly increased risk (HR 1.91; $95 \%$ CI $0.45,8.22$ ) compared to light drinkers (data not shown). We also observed an increased 
Table 2 Average weekly alcohol consumption category (average of 1975 and 1981), binge drinking status (1981), and prostate cancer risk and mortality (HR, 95 \% CI), Older Finnish Twin Cohort, 1981-2012

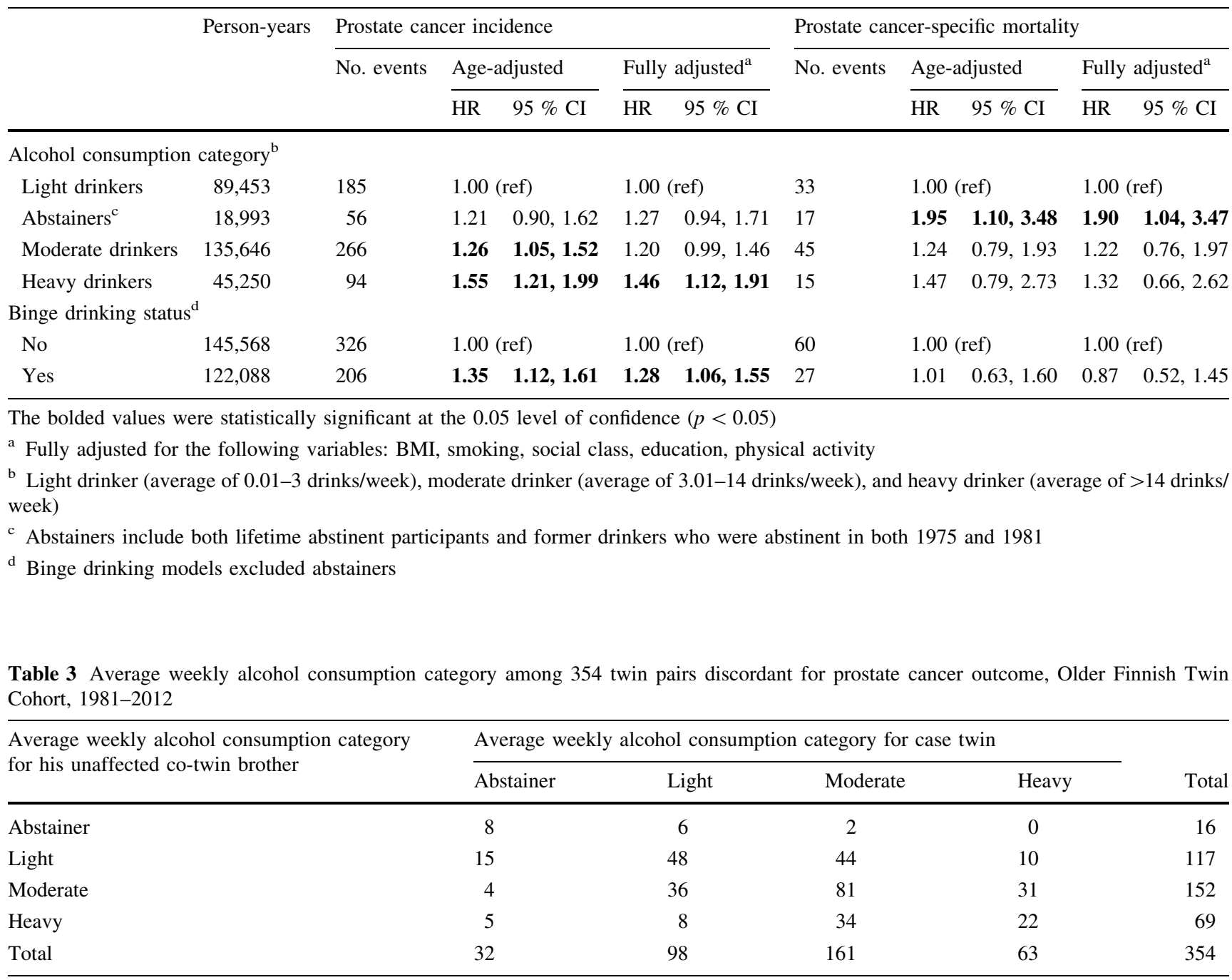

risk of mortality among moderate (HR 1.22; $95 \%$ CI 0.76 , 1.97) and heavy drinkers (HR 1.32; $95 \%$ CI 0.66, 2.62) compared to light drinkers.

Within discordant twin pairs, binge drinkers were also at a higher risk of mortality compared to their non-binge drinker brothers, although confidence intervals were wide and included the null (Table 5).

No significant interactions between alcohol consumption and smoking status were found $\left(P_{\text {interaction }}>0.4\right.$; data not shown).

\section{Alcohol-related cause of death}

The proportion of deaths due to alcohol-related causes in each category of cumulative average alcohol consumption was as follows: $0.4 \%$ of abstainers, $0.8 \%$ of light drinkers, $3.8 \%$ of moderate drinkers, and $10.4 \%$ of heavy drinkers. The hazard ratios of alcohol-related death were 1.75 for light drinkers (95\% CI 0.22, 13.96), 8.19 for moderate drinkers (95\% CI 1.14, 58.75), and 39.18 for heavy drinkers (95\% CI 5.51, 278.53) (data not shown). These findings provide support that participants' alcohol consumption category remained stable over time.

\section{Discussion}

Our study provided the unique opportunity to investigate the association between midlife average weekly alcohol intake, binge drinking patterns, and future prostate cancer 
Table 4 Weekly alcohol consumption category ${ }^{\mathrm{a}}$ and prostate cancer risk and mortality: pairwise analyses (HR, $95 \%$ CI), Older Finnish Twin Cohort, 1981-2012

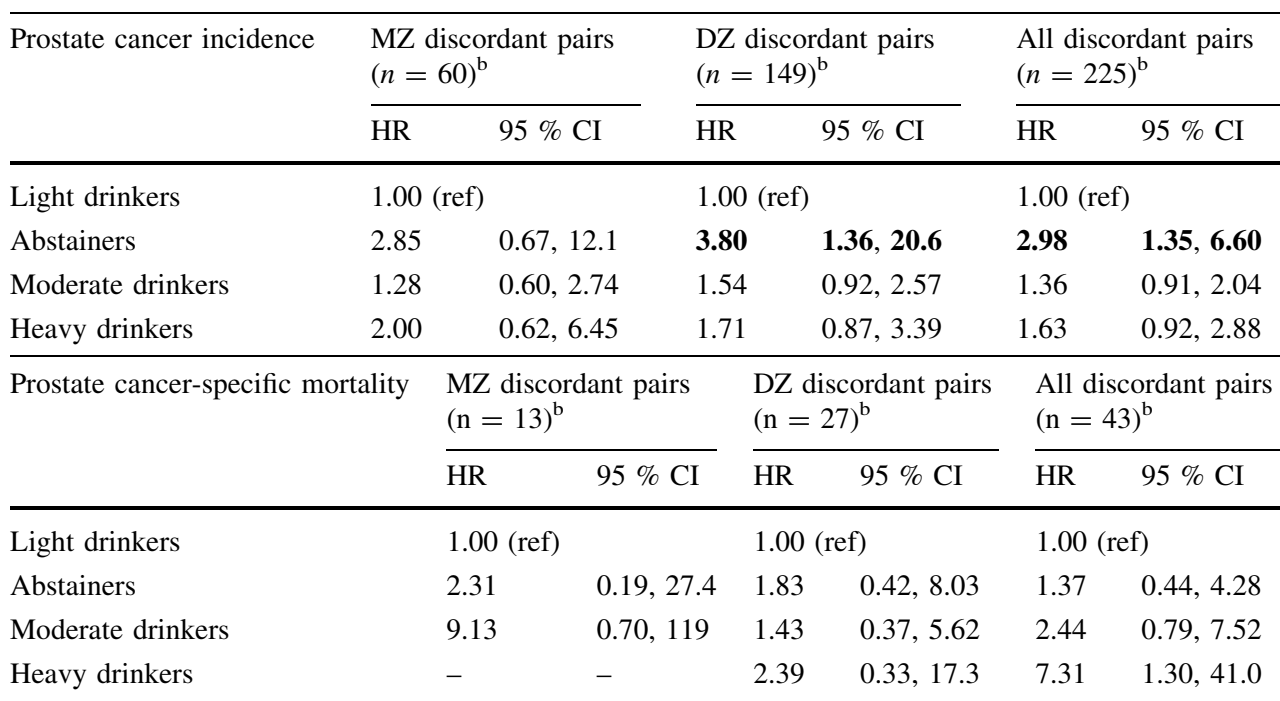

The bolded values were statistically significant at the 0.05 level of confidence $(p<0.05)$

${ }^{a}$ Light drinker (average of 0.01-3 drinks/week), moderate drinker (average of 3.01-14 drinks/week), and heavy drinker (average of $>14$ drinks/week)

b Number of twin pairs discordant for weekly alcohol consumption category and time to event (i.e., time to prostate cancer diagnosis among twin pairs concordant for prostate cancer outcome, and time to event vs. death or end of follow-up among twin pairs discordant for prostate cancer outcome)

\begin{tabular}{|c|c|c|c|c|c|c|}
\hline \multirow[t]{2}{*}{ Prostate cancer incidence } & \multicolumn{2}{|c|}{$\begin{array}{l}\text { MZ discordant pairs } \\
(n=32)^{\mathrm{a}}\end{array}$} & \multicolumn{2}{|c|}{$\begin{array}{l}\text { DZ discordant pairs } \\
(n=72)^{\mathrm{a}}\end{array}$} & \multicolumn{2}{|c|}{$\begin{array}{l}\text { All discordant pairs } \\
(n=114)^{\mathrm{a}}\end{array}$} \\
\hline & HR & $95 \% \mathrm{CI}$ & HR & $95 \% \mathrm{CI}$ & HR & $95 \% \mathrm{CI}$ \\
\hline Binge drinking status, yes versus no & 1.78 & $0.79,4.02$ & 1.46 & $0.87,2.45$ & 1.47 & $0.96,2.25$ \\
\hline \multirow[t]{2}{*}{ Prostate cancer-specific mortality } & \multicolumn{2}{|c|}{$\begin{array}{l}\text { MZ discordant pairs } \\
(n=3)^{\mathrm{a}}\end{array}$} & \multicolumn{2}{|c|}{$\begin{array}{l}\text { DZ discordant pairs } \\
(n=10)^{\mathrm{a}}\end{array}$} & \multicolumn{2}{|c|}{$\begin{array}{l}\text { All discordant pairs } \\
(n=14)^{\mathrm{a}}\end{array}$} \\
\hline & HR & $95 \% \mathrm{CI}$ & HR & $95 \% \mathrm{CI}$ & HR & $95 \% \mathrm{CI}$ \\
\hline Binge drinking status, yes versus no & 3.00 & $0.31,28.8$ & 1.25 & $0.34,4.65$ & 1.80 & $0.60,5.37$ \\
\hline
\end{tabular}

Table 5 Binge drinking status and prostate cancer risk and mortality: pairwise analyses (HR, $95 \%$ CI), Older Finnish Twin Cohort, 1981-2012 risk and mortality while controlling for potential familial confounding (genetics and shared early environment) in a large population of male twins. To the best of our knowledge, this study is the first of its kind to leverage co-twin analyses in the investigation of this association. Altogether, our data support the hypothesis that heavy regular alcohol intake and binge drinking patterns are associated with an increased risk of prostate cancer. They also suggest that abstinence may be associated with an increased risk of prostate cancer-specific mortality compared to light regular alcohol consumption. Our co-twin analyses suggest that heavy regular alcohol consumption as well as binge drinking may be associated with a higher risk of prostate cancer incidence and mortality independent of familial and genetic factors. However, this finding should be interpreted with caution given the small number of discordant pairs in the co-twin analyses.

There is biologic rationale that heavy regular or binge drinking of alcohol may increase prostate cancer risk via production of carcinogenic metabolites such as acetaldehyde, promotion of oxidative stress, enhancement of carcinogen solubility and absorption, and inhibition of detoxification and DNA methylation pathway enzymes [2, 18]. Conversely, high alcohol intake may lower testosterone levels - an altered hormonal profile that might be expected to decrease prostate cancer risk [18]. These physiologic changes may impact different stages of prostate carcinogenesis. It has been suggested that increased 
oxidative stress may adversely impact early stages, while altered hormonal profiles may favorably impact later promotion [18]. Consistent with this hypothesis, in the present study we found that heavy regular and binge drinking had a larger impact on prostate cancer incidence than on mortality. The biologic impact of alcohol is complex, and further investigation is needed to explore how the balance between its potentially adverse and protective effects varies by exposure level and how this impacts different stages of prostate carcinogenesis and grades of disease.

Despite the biologic plausibility of an association between alcohol and prostate cancer, the epidemiologic evidence has been inconsistent. A meta-analysis of 50 case-control and 22 cohort studies reported a modest association between alcohol intake and total prostate cancer risk (RR 1.05 [95\% CI 1.02, 1.09] for light drinkers (0-7 drinks/week), RR 1.06 [95\% CI 1.01, 1.11] for moderate drinkers (7-28 drinks/week), and RR 1.08 [95\% CI $0.97,1.20$ ] for heavy drinkers ( $\geq 28$ drinks/week); reference $=$ non/occasional drinkers) [11]. A more recent meta-analysis of 23 case-control and 20 cohort studies reported similar estimates for the association between alcohol consumption and prostate cancer risk (RR 1.04 [95 \% CI 1.01, 1.08] for light drinkers, RR 1.06 [1.01, 1.11] for moderate drinkers, and RR 1.09 [95 \% CI 0.98, 1.21] for heavy drinkers; reference $=$ non/occasional drinkers) [1]. Of these studies, 39 reported on prostate cancer incidence and 4 reported on mortality as the outcome of interest. Authors of this meta-analysis considered as light, moderate, and heavy drinking every interval of alcohol intake whose midpoint was, respectively, $\leq 12.5$, $\leq 50$, and $>50 \mathrm{~g} /$ day of alcohol. Trends of increasing prostate cancer risk across increasing levels of alcohol consumption have been reported [1,11], but the statistical significance of point estimates is typically lost at the highest levels of intake. In the present study, we found a significant trend of increasing prostate cancer risk with increasing average weekly alcohol consumption as well as a significant association between heavy regular drinking and prostate cancer risk. Furthermore, this association was of a stronger magnitude than the aforementioned estimates from meta-analyses [1, 11].

We also found that binge drinking consumption patterns were associated with a significantly increased risk of prostate cancer. Others have similarly found a positive association between infrequent consumption of large amounts of alcohol and prostate cancer risk [18, 26]. These findings highlight the importance of accounting for both frequency and quantity of alcohol intake, as different drinking patterns may be associated with different physiologic effects and implications for prostate cancer risk and mortality.

The inconsistency across epidemiologic reports might be rooted in a variety of factors. Case-control studies are sensitive to recall and selection bias, alcohol intake in many study populations is often limited to a narrow range, and investigators utilize varying methods of reporting alcohol consumption (i.e., different units of alcohol per duration of time, different reference groups). Individual levels of alcohol consumption vary considerably over time, but many studies have been based on alcohol intake assessed at a single time point. Finally, ecological studies report major differences in prostate cancer rates around the world, suggesting that environmental factors may play a role in the development of this disease [27]. Estimates may therefore be affected by residual confounding by environmental factors that have yet to be identified with certainty.

The unique nature of our study population and analytic approach allowed us to address these limitations. The prospective, population-based design of our study minimizes the likelihood of recall and selection bias. The standardized incidence ratio of prostate cancer in our cohort compared to Finnish Cancer Registry data was 1.00 (95\% CI 0.92-1.08). The distribution of alcohol intake in this population was broad, binge drinking rates were high, and we were able to leverage data assessed at two different time points to account for variation in midlife drinking habits over follow-up. We also repeated analyses using abstinent men as the reference group for comparison to other studies (Supplementary Tables 1 and 4). Co-twin analyses allowed us to account for the potentially confounding effects of genetics and shared early environment, since each matched set consisted of a twin pair. Our findings suggest that non-familial environmental factors (unique environmental factors as well as direct causal associations) are at play in the associations between heavy regular drinking, binge drinking, and prostate cancer risk and mortality, given the consistently elevated risk estimates across all discordant pairs compared to individualbased analyses. These results could also be due to chance despite our large overall study population; the number of discordant twin pairs was insufficient to supply the power needed to confirm these findings with statistical confidence. Thus, we also cannot exclude the possibility of some familial confounding due to shared environmental and genetic factors. These could be addressed with Mendelian randomization studies that use genetic variants associated with alcohol use and prostate cancer risk as instrumental variables, as has been conducted for the alcohol-coronary heart disease association [28].

Important strengths of our study include its prospective design, long duration of follow-up, large population-based sample, complete and reliable outcome data obtained through registry linkage, high questionnaire response rate with two assessments (84-89\%), and detailed assessment of socio-demographic, psychosocial, and health factors. In addition to examining total prostate cancer risk, we 
examined risk of prostate cancer-specific mortality, which reflects the most aggressive prostate tumors. Several limitations should be noted. The low number of discordant twin pairs available for the co-twin analyses did not provide sufficient statistical power to draw strong conclusions on familial confounding. Also, the Caucasian background of this Finnish population may limit the generalizability of these findings to more diverse groups of men. Further, we cannot rule out the possibility that the incidence findings might be partially explained by prostate-specific antigen (PSA) testing. However, we speculate that PSA testing is inversely associated with heavy drinking and positively associated with prostate cancer, such that a lack of adjustment for PSA testing would be expected to bias risk estimates toward the null. In addition, routine PSA testing among asymptomatic men was not common in Finland during the study period [29], and prostate cancer cases in Finland tend to be more aggressive at diagnosis than in the USA [30, 31]. Cases are thus expected to be clinically relevant. Lastly, both underreporting of alcohol consumption and changes over time are expected to be non-differential with respect to prostate cancer outcome since these data were collected prior to diagnosis. This type of potential exposure misclassification would therefore, if anything, bias risk estimates toward the null. Our results on the risk of alcohol-related death by alcohol consumption category support the stability of alcohol consumption category over time.

\section{Conclusion}

In this prospective, population-based cohort study of Finnish male twins with a median 30 years of follow-up, we found that heavy regular alcohol consumption and binge drinking patterns were associated with an increased risk of prostate cancer incidence, while abstinence was associated with an increased risk of prostate cancer-specific mortality compared to light alcohol consumption. Co-twin analyses suggest that alcohol consumption may directly impact prostate cancer risk, although they do not exclude the possibility that the association may at least partially depend on familial factors (genetics and shared early environment). Future studies are needed to further explore the biologic effects of alcohol on the prostate to elucidate how different levels of exposure impact different stages of prostate carcinogenesis.

Acknowledgments We are grateful to Kauko Heikkilä, Ph.Lic, for assistance in database management.

Grants JK is supported by the Academy of Finland (Grants 265240 and 263278). SCM is supported by the National Cancer Institute at the National Institutes of Health Training Grant NIH T32 CA09001.

\section{Compliance with ethical standards}

Conflict of interest The authors declare that they have no conflicts of interest.

\section{References}

1. Bagnardi V, Rota M, Botteri E, Tramacere I, Islami F, Fedirko V et al (2015) Alcohol consumption and site-specific cancer risk: a comprehensive dose-response meta-analysis. $\mathrm{Br} \mathrm{J}$ Cancer 112(3):580-593

2. International Agency for Research on Cancer (2010) IARC monographs on the evaluation of carcinogenic risks to humans, vol 96, alcohol consumption and ethyl carbamate. IARC, Lyon

3. World Cancer Research Fund/American Institute for Cancer Research (2007) Food, nutrition, physical activity, and the prevention of cancer: a global perspective. AICR, Washington

4. Eriksson CJ (2015) Genetic-epidemiological evidence for the role of acetaldehyde in cancers related to alcohol drinking. Adv Exp Med Biol 815:41-58

5. Jensen OM, Paine SL, McMichael AJ, Ewertz M (1996) Alcohol. In: Schottenfeld D, Fraumeni JF (eds) Cancer epidemiology and prevention, 2nd edn. Oxford University Press, New York, pp 290-318

6. Scoccianti C, Cecchini M, Anderson AS, Berrino F, BoutronRuault MC, Espina C, et al. European code against cancer 4th edition: alcohol drinking and cancer. Cancer Epidemiol. Published online ahead of print 2015. doi:10.1016/j.canep.2015.01.007

7. Seitz HK, Mueller S (2015) Alcohol and cancer: an overview with special emphasis on the role of acetaldehyde and cytochrome P450 2E1. Adv Exp Med Biol 815:59-70

8. Breslow RA, Weed DL (1998) Review of epidemiologic studies of alcohol and prostate cancer: 1971-1996. Nutr Cancer 30(1):1-13

9. Dennis LK, Hayes RB (2001) Alcohol and prostate cancer. Epidemiol Rev 23(1):110-114

10. Fillmore KM, Chikritzhs T, Stockwell T, Bostrom A, Pascal R (2009) Alcohol use and prostate cancer: a meta-analysis. Mol Nutr Food Res 53(2):240-255

11. Rota M, Scotti L, Turati F, Tramacere I, Islami F, Bellocco R et al (2012) Alcohol consumption and prostate cancer risk. Eur J Cancer Prev 21(4):350-359

12. Albertsen K, Grønbaek M (2002) Does amount or type of alcohol influence the risk of prostate cancer? Prostate 52(4):297-304

13. Crispo A, Talamini R, Gallus S, Negri E, Gallo A, Bosetti C et al (2004) Alcohol and the risk of prostate cancer and benign prostatic hyperplasia. Urology 64(4):717-722

14. Watters JL, Park Y, Hollenbeck A, Schatzkin A, Albanes D (2010) Alcoholic beverages and prostate cancer in a prospective US cohort Study. Am J Epidemiol 172(7):773-780

15. McGregor SE, Courneya KS, Kopciuk KA, Tosevski C, Friedenreich CM (2013) Case-control study of lifetime alcohol intake and prostate cancer risk. Cancer Causes Control 24(3):451-461

16. Gong Z, Kristal AR, Schenk JM, Tangen CM, Goodman PJ, Thompson IM (2009) Alcohol consumption, finasteride, and prostate cancer risk: results from the Prostate Cancer Prevention Trial. Cancer 115(16):3661-3669

17. Hayes RB, Brown LM, Schoenberg JB, Greenberg RS, Silverman DT, Schwartz AG (1996) Occupational risk factors and prostate cancer in US blacks and whites. Am J Epidemiol 143(7):692-697

18. Platz EA, Leitzmann MF, Rimm EB, Willett WC, Giovannucci E (2004) Alcohol intake, drinking patterns, and risk of prostate 
cancer in a large prospective cohort study. Am $\mathrm{J}$ Epidemiol 159(5):444-453

19. Putnam SD, Cerhan JR, Parker AS, Bianchi GD, Wallace RB, Cantor KP et al (2000) Lifestyle and anthropometric risk factors for prostate cancer in a cohort of Iowa men. Ann Epidemiol 10(6):361-369

20. Sesso HD, Paffenbarger RS, Lee IM (2001) Alcohol consumption and risk of prostate cancer: The Harvard Alumni Health Study. Int J Epidemiol 30(4):749-755

21. Tonnesen H, Moller H, Andersen JR, Jensen E, Juel K (1994) Cancer morbidity in alcohol abusers. Br J Cancer 69:327-332

22. Velicer C, Kristal A, White E (2009) Alcohol use and the risk of prostate cancer: results from the VITAL cohort study. Nutr Cancer 56(1):50-56

23. Sarna S, Kaprio J, Sistonen P, Koskenvuo M (1978) Diagnosis of twin zygosity by mailed questionnaire. Hum Hered 28(4):241-254

24. National Institute on Alcohol Abuse and Alcoholism (NIHAAA). Drinking levels defined. http://www.niaaa.nih.gov/alcohol-health/ overview-alcohol-consumption/moderate-binge-drinking. Accessed 29 July 2015

25. Kujala UM, Kaprio J, Koskenvuo M (2002) Modifiable risk factors as predictors of all-cause mortality: the roles of genetics and childhood environment. Am J Epidemiol 156(11):985-993
26. Baglietto L, Severi G, English DR, Hopper JL, Giles GG (2006) Alcohol consumption and prostate cancer risk: results from the Melbourne collaborative cohort study. Int J Cancer 119(6):1501-1504

27. Haas GP, Sakr WA (1997) Epidemiology of prostate cancer. CA Cancer J Clin 47(5):273-287

28. Holmes MV, Dale CE, Zuccolo L, Silverwood RJ, Gou Y, Ye Z et al (2014) Association between alcohol and cardiovascular disease: Mendelian randomization analysis based on individual participant data. BMJ 349:g4164

29. Pogodin-Hannolainen D, Juusela H, Tammela TL, Ruutu M, Aro J, Määttänen L et al (2011) Prostate cancer screening: a survey of attitudes and practices among Finnish physicians in 1999 and 2007. J Med Screen 18:46-49

30. Andriole GL, Crawford ED, Grubb RL, Buys SS (2009) Mortality results from a randomized prostate-cancer screening trial. N Engl J Med 360:1310-1319

31. Mäkinen T, Tammela TLJ, Hakama M, Stenman UH, Rannikko S, Aro J et al (2003) Tumor characteristics in a population-based prostate cancer screening trial with prostate-specific antigen. Clin Cancer Res 9:2435-2439 